\section{Integrative Medicine \\ International}

\title{
Ginkgo biloba Extract Inhibits Platelet Activation via Inhibition of Akt
}

\author{
Ye Shiyong Xiang Yijia Zhong Peng Liu Chong Hu Wuming \\ Lv Linchun Zeng Chunlai \\ The Fifth Affiliated Hospital of Wenzhou Medical College, Lishui Central Hospital, \\ Lishui, China
}

\author{
Key Words \\ Ginkgo biloba extract $\cdot$ PI3K/Akt $\cdot$ Platelets
}

\begin{abstract}
Ginkgo biloba extract (GBE) contains flavone glycosides and terpenoids. It can modify vasomotor function, reduce the adhesion of blood cells to the endothelium, and inhibit the activation of platelets, and therefore plays an important role in the treatment of a variety of cardiovascular and neurological disorders. However, the molecular mechanism underlying its significant anticoagulation activity is largely unknown. In this study, we found that GBE adenosine diphosphate, collagen, and U4 (U46619) induced platelet aggregation in both plateletrich plasma and washed platelets. GBE could reduce the adhesion of platelets to a collagencoated surface and platelet spreading on fibrinogen. The treatment with GBE significantly inhibited the phosphorylation of Akt, suggesting that its inhibition of platelet activation might be mediated by attenuation of the PI3K/Akt pathway.

(C) 2015 S. Karger AG, Basel
\end{abstract}

\section{Introduction}

When a blood vessel is injured, platelets adhere to the exposed subendothelial matrix by binding to von Willebrand factor (VWF) and collagen. Then, activated platelets secrete mediators such as thromboxane $\mathrm{A} 2$ and adenosine diphosphate (ADP) to activate more platelets to form aggregates. Finally, postaggregation events take place to enable the perpetuation of thrombosis [1]. Phosphoinositide 3-kinase (PI3K) plays a key role during platelet activation. 
Akt (also known as protein kinase B), an important downstream effector of PI3K and its phosphorylation, can be used as a marker of the activation of the PI3K pathway. Akt is a 57-kDa serine/threonine kinase, which has at least 3 different Akt isoforms identified in humans, named Akt1, Akt2, and Akt3. The major Akt subtype in human platelets is Akt1. Upon activation of PI3K, Akt undergoes phosphorylation at its regulatory threonine and serine phosphorylation sites (Akt-1, Thr308/Ser473; Akt-2, Thr309/Ser474) [2].

Hyperactivity of platelets can potentially lead to myocardial infarction or stroke, the major causes of death in many industrialized countries [3]. Antiplatelet medicine has been heralded as a major breakthrough in clinical intervention. Some antiplatelet drugs, such as the glycoprotein $\alpha \mathrm{IIb} / \beta 3$ inhibitor ReoPro, the cyclooxygenase inhibitor aspirin, and the P2Y12 inhibitor clopidogrel, have significantly changed the outcome in patients suffering from cardiovascular and cerebral diseases. However, a significant number of patients have aspirin/clopidogrel resistance. Novel antiplatelet/antithrombotic medicine is still needed and under intense investigation, including natural compounds or effective ingredients in medical herbs.

Ginkgo biloba, also named living fossil, refers to one kind of tree cultivated in China. Its clinical use can date back to as early as the origin of Chinese medicine, and various formulations of $G$. biloba have already become the best-selling herbal remedies in the whole world. G. biloba extract (GBE), which contains flavone glycosides and terpenoids, has also been proven to have preventive and therapeutic effects for cardiovascular diseases. However, the underlying molecular mechanisms of GBE still remain largely unknown [4]. Here, we investigated the molecular signaling transduction pathway underlying the inhibition of GBE on platelet activation. We demonstrated, for the first time, that GBE significantly inhibited the phosphorylation of Akt, suggesting that its inhibition of platelet activation might be mediated by attenuation of the PI3K/Akt pathway.

\section{Materials and Methods}

\section{Study Subjects}

This study was approved by the Ethics Committee of Lishui Central Hospital. All healthy subjects (aged 20-29 years) were recruited from the staff and students at the Wenzhou Medical College. Informed consent was obtained from all volunteers.

Chemicals

GBE was purchased from Zhejiang CONBA Pharmaceutical Co. Ltd. and dissolved in DMSO (dimethyl sulfoxide). HEPES, ADP, bovine serum albumin, and apyrase were all purchased from Sigma (St. Louis, Mo., USA). Collagen was from Chrono-Log Corp. (Havertown, Pa., USA). The pan-PI3K inhibitor LY294002 was from Calbiochem-Novabiochem Corp (San Diego, Calif., USA). R-phycoerythrin-conjugated anti-CD62 mAb AC1.2 (Becton Dickinson) was monitored for platelet P-selectin expression.

\section{Human Blood Collection and Preparation of Washed Platelets and Red Blood Cells}

Whole blood was obtained by venous puncture from healthy nonsmoking human donors (aged 20-29 years) who had not taken any medication during at least 2 weeks preceding venipuncture. Blood was drawn without stasis into tubes containing 3.8\% 1:9 (v/v) sodium citrate. Blood was anticoagulated with acid citrate dextrose (ACD; $39 \mathrm{~mm}$ citric acid, $75 \mathrm{~mm}$ sodium citrate, $135 \mathrm{~mm}$ dextrose, $\mathrm{pH}$ 6.5), and centrifuged at $150 \mathrm{~g}$ for 20 min to collect the platelet-rich plasma (PRP). After being diluted 3-fold by ACD containing $0.1 \mathrm{U} / \mathrm{ml}$ apyrase, the PRP was then centrifuged for $10 \mathrm{~min}$ at $800 \mathrm{~g}$. The platelet pellet was then resuspended in Tyrode's buffer ( $2 \mathrm{~mm} \mathrm{KCl}, 1 \mathrm{~mm} \mathrm{MgCl}, 137 \mathrm{~mm} \mathrm{NaCl}, 12 \mathrm{~mm} \mathrm{NaHCO}_{3}, 0.34 \mathrm{mM} \mathrm{Na}_{2} \mathrm{HPO}_{4}, 5.5 \mathrm{~mm}$ glucose, $0.35 \%$ bovine serum albumin, and $5 \mathrm{mM} \mathrm{HEPES}$ ) and adjusted to a concentration of $250 \times 10^{6}$ platelets $/ \mathrm{ml}$. $\mathrm{CaCl}_{2}(2 \mathrm{~mm})$ was added prior to the agonist stimulation. For the isolation of washed red blood cells, the remaining blood after PRP collection was washed with Tyrode's buffer containing ACD and centrifuged 3 times at $800 \mathrm{~g}$ for $10 \mathrm{~min}$ to remove plasma proteins. 


\section{Control Group}

Control group was the DMSO group. Compared with the drug of the experimental group, the DMSO group had the same concentration of DMSO.

Flow Cytometry

Flow cytometric analyses of platelet P-selectin expression have been described previously [5]. R-phycoerythrin-CD62P fluorescence was monitored to obtain the percentage of P-selectin-positive platelets.

\section{Platelet Aggregation}

Platelet aggregation was measured using a 4-channel Chrono-Log aggregometer. PRP was preincubated with vehicle or $\operatorname{GBE}(2,1$, and $0.2 \mathrm{mg} / \mathrm{ml})$ for $10 \mathrm{~min}$ at $37^{\circ} \mathrm{C}$ without stirring. The agonists were then added by stirring at $900 \mathrm{rpm}[6]$.

\section{Platelet Adhesion on Collagen-Coated Surface under Flow Conditions}

The experiment was performed as described previously, with minor modifications [7]. Briefly, glass coverslips $(24 \times 50 \mathrm{~mm})$ were cleaned with a dichromic acid solution overnight, rinsed with distilled water, and dried before coating. At a density of $0.08 \mu \mathrm{g} / \mathrm{cm}^{2}$, collagen was coated on coverslips at $4{ }^{\circ} \mathrm{C}$ overnight. The flow chamber was then assembled. A peristaltic pump (Masterflex L/S; Cole-Parmer, Vernon Hills, Ill., USA) was used to aspirate blood through the flow chamber. The fluorescence dye calcein $(10 \mu \mathrm{g} / \mathrm{ml}$; Molecular Probes, Eugene, Oreg., USA) was added to the washed platelets. After 20 min of incubation, calcein-labeled platelets were resuspended and mixed with washed red blood cells to form reconstituted blood with a platelet concentration of $200 \times 10^{5} / \mathrm{ml}$ and a hemocrit level of $45 \%$. The reconstituted blood was incubated at $37^{\circ} \mathrm{C}$ for 10 min with or without GBE ( $2 \mathrm{mg} / \mathrm{ml})$ or LY294002 $(25 \mu \mathrm{M})$. Blood was perfused over the coverslip for $5 \mathrm{~min}$ at a shear rate of 1,000/s. The perfusion was live-monitored with a fluorescence microscope (Nikon TE-2000S; Nikon, Melville, N.Y., USA). After the termination of perfusion, the flow chamber was washed with phosphate-buffered saline (PBS) for 2 min to remove nonadherent cells. The images from at least 10 independent fields per flow experiment were recorded and analyzed by using IMAGE-PRO PLUS software (Media Cybernetics, Atlanta, Ga., USA).

\section{Platelet Spreading on Fibrinogen}

$10 \mu \mathrm{g} / \mathrm{ml}$ fibrinogen (in $0.1 \mathrm{M} \mathrm{NaHCO}_{3}, \mathrm{pH} 8.3$ ) was coated on glass coverslips $\left(24 \times 50 \mathrm{~mm}\right.$ ) at $4^{\circ} \mathrm{C}$ overnight. Washed platelets $\left(2 \times 10^{7} / \mathrm{ml}\right)$ preincubated with $2 \mathrm{mg} / \mathrm{ml} \mathrm{GBE}$ or $25 \mu \mathrm{M} \mathrm{LY} 294002\left(10 \mathrm{~min}\right.$ at $\left.37^{\circ} \mathrm{C}\right)$ or without inhibitors were allowed to spread on the fibrinogen-coated surfaces for $90 \mathrm{~min}$ at $37^{\circ} \mathrm{C}$. After 3 washes with PBS, the cells were fixed, permeabilized, and stained with fluorescein-labeled phalloidin, respectively. Adherent platelets were monitored with an inverted fluorescence microscope (Nikon TE-2000S). Images were acquired using a Nikon DS-2MBWc-U1 CCD camera. The spreading area of individual platelets was analyzed using IMAGE-PRO PLUS software (Media Cybernetics) [8].

\section{Immunoblotting}

Aliquots of washed platelets $\left(0.25 \mathrm{ml} ; 250 \times 10^{6} / \mathrm{ml}\right)$ preincubated with vehicle or GBE ( $\left.2 \mathrm{mg} / \mathrm{ml}\right)$ for 10 min were stimulated with agonists at $37^{\circ} \mathrm{C}$. The reaction was split by sodium dodecyl sulfate (SDS) sample buffer (2\% SDS, 10\% glycerol, $62.5 \mathrm{~mm}$ Tris- $\mathrm{HCl}, 50 \mathrm{~mm}$ dithiothreitol, $0.1 \%$ bromophenol blue; $\mathrm{pH}$ 6.8). Samples were boiled for $5 \mathrm{~min}$ at $95^{\circ} \mathrm{C}$ before being stored at $-20^{\circ} \mathrm{C}$. Sample aliquots ( $\left.40 \mu \mathrm{l}\right)$ were loaded, and the proteins were separated on $10 \%$ SDS polyacrylamide gel electrophoresis gel. The proteins were then transferred to a polyvinylidene fluoride membrane and subjected to Western blotting. Akt and phospho-Akt were detected using the anti-Akt (total) antibody (catalog No. 9272) and the anti-phospho-Akt (Ser473/474) antibody (clone 587F11), respectively. Similarly, the mitogen-activated protein kinase (MAPK) p38 (catalog No. 9212) and phospho-p38 (28B10) and the tyrosine kinase Src (L4A1) and phospho-Src (Tyr416) were detected using corresponding antibodies (all from Cell Signaling Technology, Beverly, Mass., USA). After incubation with the corresponding secondary antibodies goat anti-rabbit IRDye 800CW and goat anti-mouse IRDye 680 (LI-COR Biosciences, Lincoln, Nebr., USA), densitometric band scanning was performed using an Odyssey infrared imaging system (LI-COR Biosciences) [9].

\section{Statistics}

The data are presented as means \pm standard errors of the mean. Individual measurements of GBE effects were compared with Wilcoxon's signed rank test. Values were considered to be statistically significant when $\mathrm{p}<0.05$. 


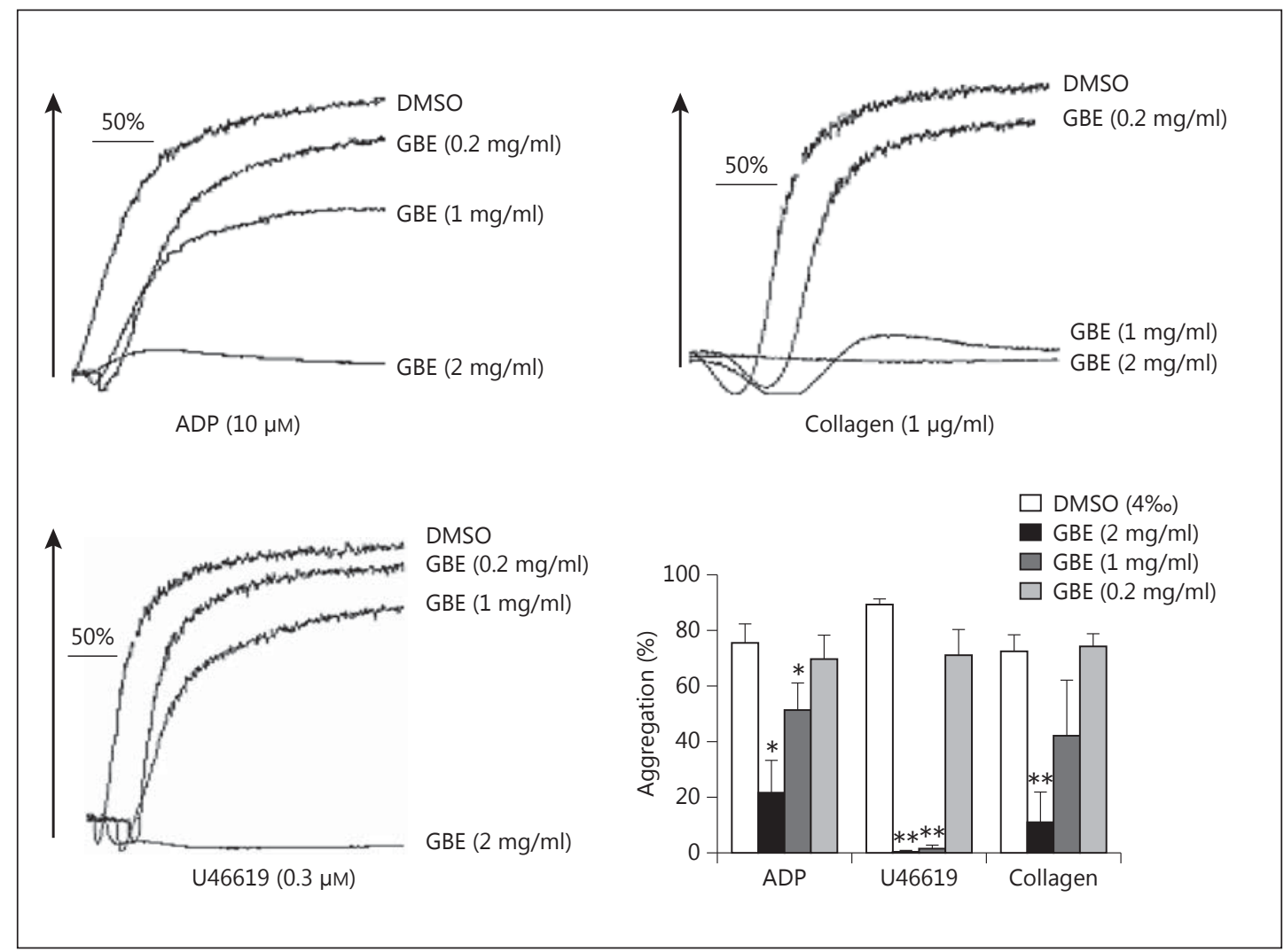

Fig. 1. GBE dose-dependently inhibited platelet aggregation induced by ADP, collagen, and U46619. GBE (2, 1 , and $0.2 \mathrm{mg} / \mathrm{ml}$ ) or DMSO (4\%) was preincubated with PRP for $10 \mathrm{~min}$. Platelet aggregation was induced by ADP $(10 \mu \mathrm{M})$, collagen $(1 \mu \mathrm{g} / \mathrm{ml})$, or U46619 $(0.6 \mu \mathrm{M})$. Means \pm standard errors of the mean of platelet aggregation percentage are plotted in the bar chart $(n=5){ }^{*} \mathrm{p}<0.05$ and ${ }^{* *} \mathrm{p}<0.01$ versus control.

\section{Results}

\section{Effects of GBE on Platelet Aggregation}

Platelets aggregate when added with different agonists coupled with the measurement of stored and releasable platelet nucleotide content. As shown in figure 1, GBE can reduce platelet aggregation in PRP induced by ADP, collagen, and U46619 in a dose-dependent manner. For example, when induced by ADP, $2 \mathrm{mg} / \mathrm{ml}$ GBE almost abolished the platelet aggregation, while 1 and $0.2 \mathrm{mg} / \mathrm{ml} \mathrm{GBE}$ just inhibited it to $51.33 \pm 9.60 \%$ and $69.67 \pm 8.51 \%$, respectively, when compared with the control $(75.33 \pm 6.89 \%)$.

\section{Influence of GBE on Agonist-Induced Single Platelet Activation}

To investigate the agonist-induced single platelet activation, we used PRP to test the expression on the platelets by flow cytometry. P-selectin, which is packaged into $\alpha$-granules, was obviously increased by ADP $(1 \mu \mathrm{M})$ and $\mathrm{U} 46619(0.6 \mu \mathrm{M})$, from $3.11 \pm 0.37 \%$ at baseline to $71.87 \pm 2.35 \%$ (fig. $2 \mathrm{a}$ ) and from $3.12 \pm 0.37 \%$ to $66.76 \pm 9.44 \%$ (fig. $2 \mathrm{~b}$ ), respectively ( $\mathrm{n}=$ 6 ; $p<0.01$ and $p<0.05$, respectively). Additionally, the DMSO-added groups were similar to the agonist groups. However, when treated with $2 \mathrm{mg} / \mathrm{ml} \mathrm{GBE}$, the levels of P-selectin expression were reduced to $6.83 \pm 1.07 \%$ (fig. $2 \mathrm{a}$ ) and $5.51 \pm 0.77 \%$ (fig. $2 \mathrm{~b}$ ). 


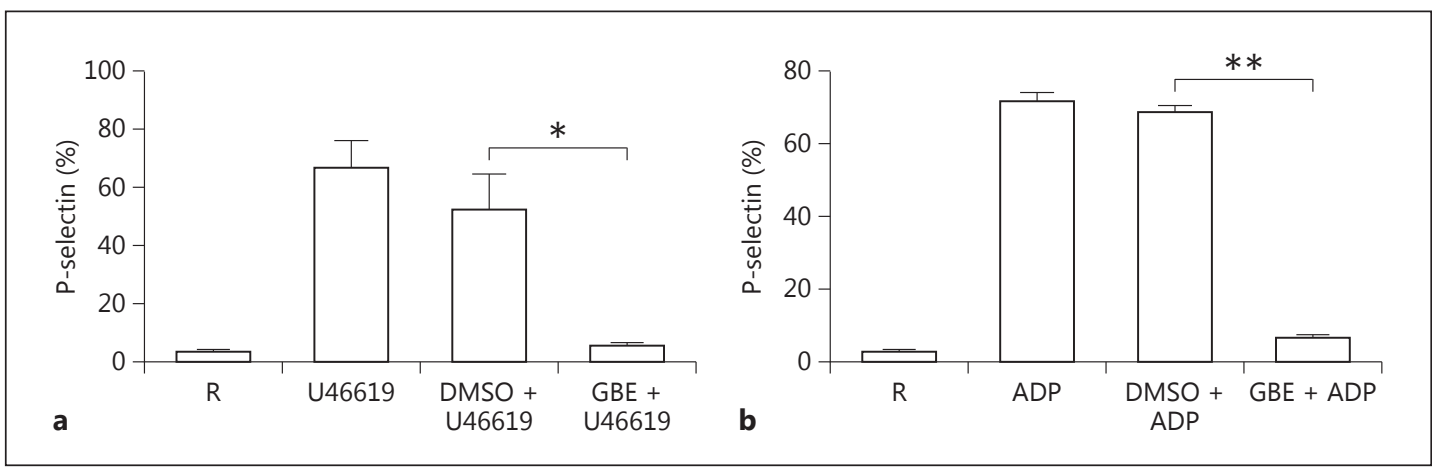

Fig. 2. GBE reduced U46619- (a) and ADP-enhanced (b) platelet P-selectin expression. Diluted PRP was preincubated with GBE ( $\left.2 \mathrm{mg} / \mathrm{ml} ; 22^{\circ} \mathrm{C} ; 10 \mathrm{~min}\right)$, DMSO $\left(4 \%\right.$; $\left.22^{\circ} \mathrm{C} ; 10 \mathrm{~min}\right)$, or vehicle in the presence of fluorescent P-selectin. Samples were then stimulated with U46619 (0.6 $\mu \mathrm{M})$ or ADP (1 $\mu \mathrm{M})$ for 10 min. P-selectin expression of single platelets was monitored by flow cytometry. Data plotted are means \pm standard errors of the mean $(n=6){ }^{*} \mathrm{p}<0.05$ and ${ }^{* *} \mathrm{p}<0.01$, without versus with GBE treatment.

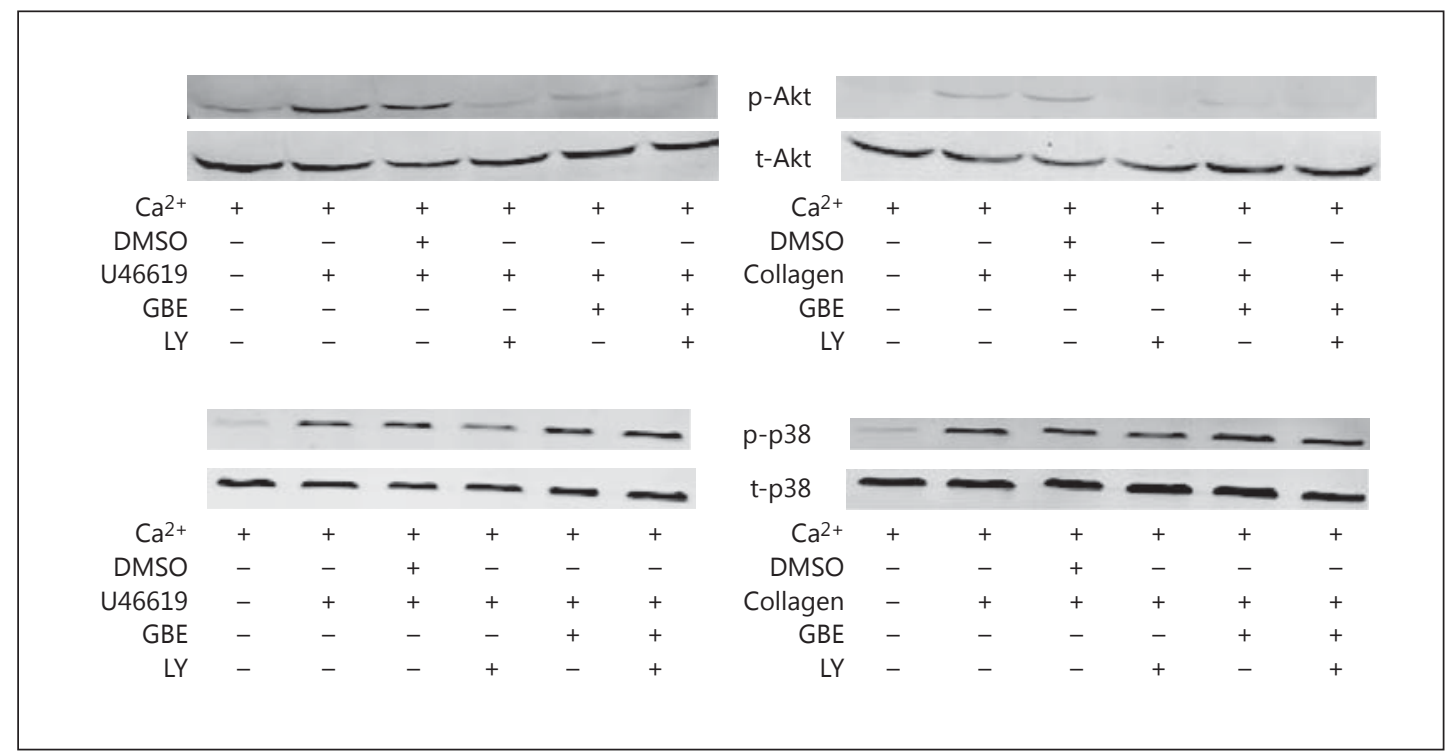

Fig. 3. GBE acted on the PI3K pathway. Washed human platelets $\left(250 \times 10^{6} / \mathrm{ml}\right)$ were preincubated with or without GBE $(2 \mathrm{mg} / \mathrm{ml})$, and then stimulated with collagen $(1 \mu \mathrm{g} / \mathrm{ml})$ or $\mathrm{U} 46619(0.6 \mu \mathrm{M})$ with stirring at 900 $\mathrm{rpm}$ in an aggregometer at $37^{\circ} \mathrm{C}$. Then, platelets were lysed and immunoblotted using the corresponding antibodies recognizing total $(\mathrm{t})$ or phosphorylated $(\mathrm{p})$ enzymes: p38, Akt and Src.

\section{Influence of GBE on Platelet Intracellular Signaling}

Three pathways play an important role for intracellular signaling targets during platelet activation: the MAPK pathway (p38 phosphorylation), the PI3K pathway (Akt ${ }^{\mathrm{Ser} 473 / 474}$ phosphorylation as the activation marker), and Src kinase activation. To investigate the possible pathway, washed human platelets were preincubated with DMSO (4\%o), GBE (2 mg/ml), or LY294002 $(25 \mu \mathrm{M})$ and then added with different agonists or not, followed by clearing for the proteins. Western blotting showed that GBE had no effect on Src or p38 phosphorylation, but markedly inhibited Akt ${ }^{\mathrm{Ser} 473 / 474}$ phosphorylation induced by both U46619 and collagen. This indicates that GBE acts on the PI3K pathway (fig. 3). 


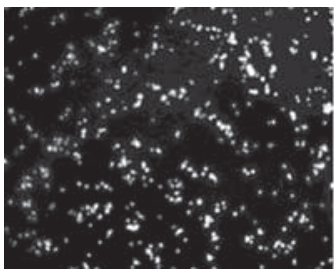

a

Control

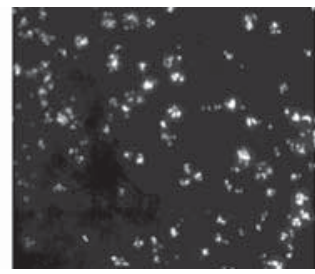

GBE

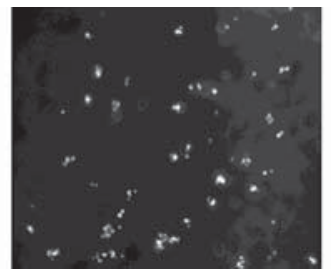

LY294002

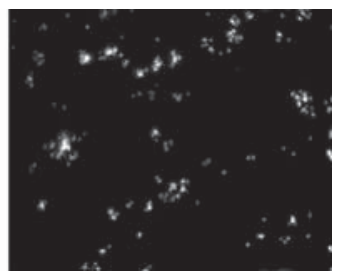

$\mathrm{GBE}+\mathrm{LY} 294002$

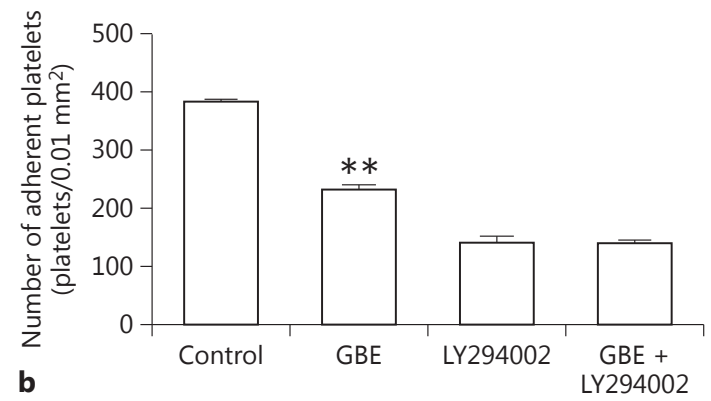

Fig. 4. Effects of GBE on platelet adhesion to collagen-coated surface. a Calcein-labeled platelets $\left(250 \times 10^{5} /\right.$ $\mathrm{ml}$ ) were added to red blood cells. The reconstituted blood was incubated for $10 \mathrm{~min}$ at $37^{\circ} \mathrm{C}$ with GBE ( $2 \mathrm{mg} / \mathrm{ml})$, DMSO (4\%o), LY294002 (25 $\mu \mathrm{M})$, and a combination of GBE and LY294002 for $10 \mathrm{~min}$ at $37^{\circ} \mathrm{C}$, respectively. The reconstituted blood was then perfused over collagen-coated $\left(0.08 \mu \mathrm{g} / \mathrm{mm}^{2}\right)$ coverslips for $5 \mathrm{~min}$ at a shear rate of $1,000 / \mathrm{s}$. PBS was washed in order to remove nonadherent cells. $\mathbf{b}$ Data plotted were means \pm standard errors of the adherent platelets per $0.01 \mathrm{~mm}^{2}(\mathrm{n}=3)$. ${ }^{* *} \mathrm{p}<0.05$ versus control.

\section{Effects of GBE on Platelet Adhesion under Flow}

Platelets can be activated by physical stress from blood flow shear and adhere to the exposed collagen surface. As for simulation, we perfused reconstituted blood over collagencoated coverslips at a shear rate of $1,000 / \mathrm{s}$, leading to platelet adhesion. The densities of adherent platelets were markedly reduced when treated with GBE and LY294002 (fig. 4). Dramatically, the combination of GBE and LY294002 did not strengthen the inhibitory effect.

\section{Effects of GBE on Platelet Spreading on Immobilized Fibrinogen}

As shown, platelet inside-out signaling such as aggregation and fibrinogen binding was inhibited by GBE. We then investigated whether GBE could affect platelet outside-in signaling, which is largely dependent on PI3K (fig. 5). GBE significantly inhibited the platelet spreading. The average surface coverage was $7.71 \pm 0.26 \mu \mathrm{m}^{2}$, reduced to $13.97 \%$ compared with the control but equal to LY2944002 with or without GBE.

\section{Discussion}

GBE has been widely used in the management of cognitive impairment in the elderly population [10] and has become one of the best-selling herbs in the world. GBE has been shown to improve mental focus in Alzheimer's disease. Recently, several groups reported possible hemorrhagic complications from G. biloba preparations [11]. It was also found that GBE could potentiate the effects of both anticoagulant and antiplatelet medications, such as 


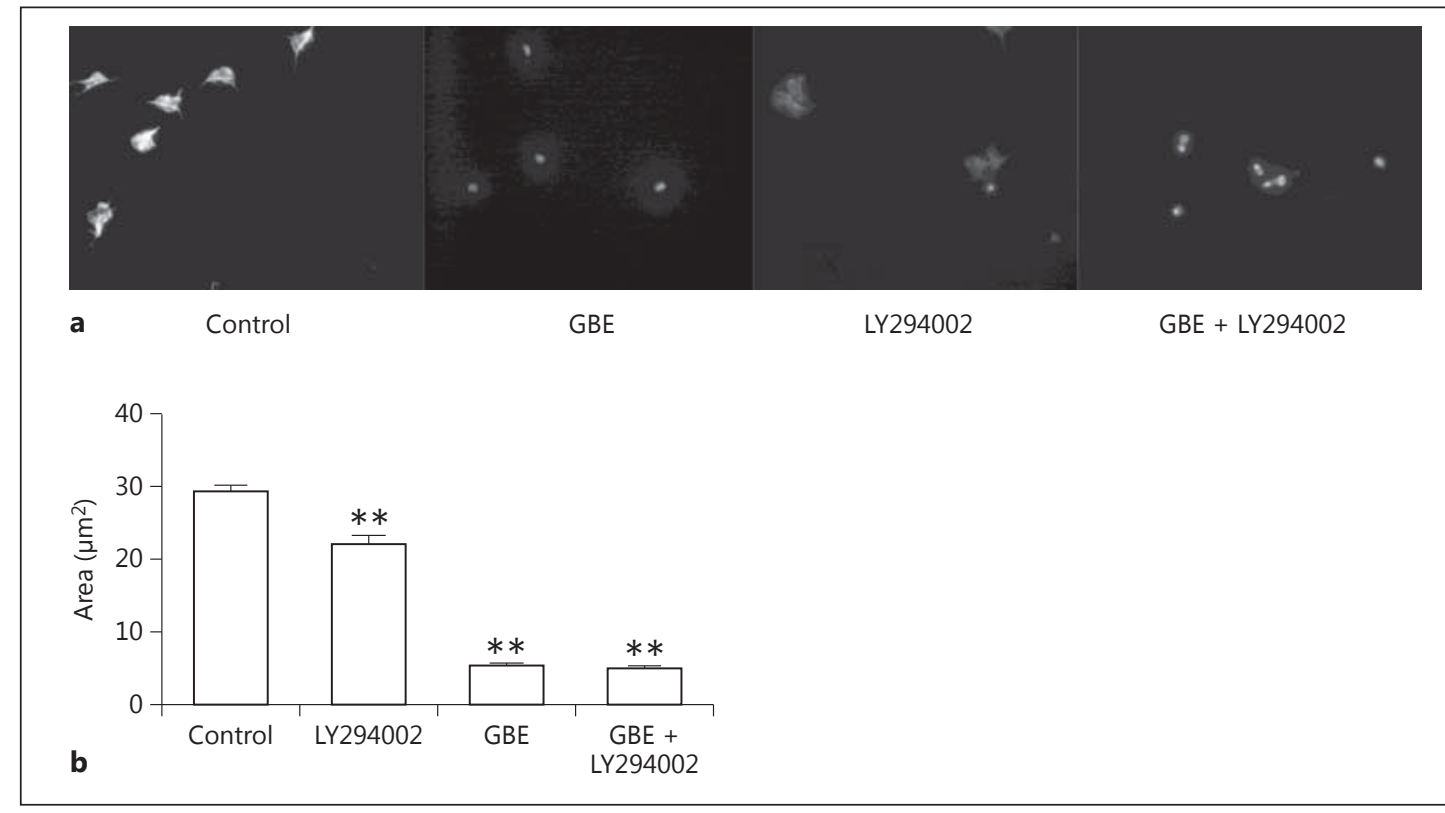

Fig. 5. GBE inhibited platelet spreading on fibrinogen surface. a Washed platelets $\left(250 \times 10^{7} / \mathrm{ml}\right)$ were incubated with GBE (2 mg/ml), DMSO (4\%o), LY294002 (25 $\mu \mathrm{M})$, and a combination of GBE and LY294002 for 10 min at $37^{\circ} \mathrm{C}$, respectively. Platelets were allowed to spread on fibrinogen-coated slides for 90 min. Platelets were subsequently labeled with fluorescein isothiocyanate-conjugated phalloidin. b Data plotted were means \pm standard errors of the average surface area of individual platelets $(n=3) .{ }^{* *} p<0.05$ versus control.

cilostazol. Combined therapy of cilostazol and GBE increased bleeding events up to 2-fold [12]. A recent study suggested a 'potentially severe interaction' between ginkgo and warfarin [13]. Therefore, exploring the mechanism underlying its significant anticoagulation activity becomes crucial.

Our study showed that GBE not only dose-dependently inhibited the aggregation of platelets caused by a pair of agonists, such as ADP and collagen, but also reduced the expression of P-selectin, a marker of platelet activation. Furthermore, we demonstrated that GBE inhibited the phosphorylation of Akt, one of the downstream molecules of PI3K, suggesting that the inhibitory effects of GBE on platelets may be mediated by inhibition of the PI3K pathway.

GBE has previously been shown to inhibit platelet aggregation in vitro and is considered an antiplatelet medicine. GBE was first found in the 1960s to be useful to improve peripheral microcirculation. The terpenoids in ginkgo were subsequently proven to be potent antagonists for platelet-activating factor. These studies led to the perception that GBE might possess clinical antiplatelet activity [14].

GBE inhibited platelet activation induced by multiple agonists, such as collagen, U46619, and ADP, suggesting that it may interact with a common signal molecule in the cascade of platelet activation. It has been reported that Akt may work through NO to suppress platelet aggregation [15-17]. Our Western blotting study showed that GBE inhibited the phosphorylation of Akt, the downstream molecule of PI3K, instead of Src (Src kinase activation) or P38 (MAPK pathway). In addition, GBE has a similar working efficacy to LY294002, an inhibitor for all types of isoforms of PI3K [18]. When we combined GBE and LY294002, there was no further reduction of adherent platelets, indicating that the inhibitory effects of GBE on platelets via PI3K inhibition are saturated. In contrast to the inhibitory effect of GBE on 
platelet aggregation and adhesion, which is mediated by the change inside the platelet signaling cascade, its inhibitory effect on platelet spreading is largely relying on a different mechanism. The engagement of $\alpha \mathrm{IIb} / \beta 3$ activates Src family kinases, which leads to the activation of Syk, Cbl-b, BTK, SLP76, and phospholipase C $\gamma 2$ (PLC $\gamma 2$ ) [19-23], promoting actin assembly, which eventually contributes to the formation of filopodia and lamellipodia [24]. As shown in our results, the inhibitory potential of GBE is much higher than LY294002, indicating that, other than Akt, GBE, with its diverse components, may also work through another mechanism to inhibit the function of platelets.

Platelet activation involves a series of rapid positive feedback loops that greatly amplify initial activation signals and allow low concentrations of agonists to induce maximal platelet responses. One important mechanism of self-amplification is the secretion of platelet granule contents such as the platelet agonist ADP after stimulation by agonists such as U46619 or collagen $[25,26]$. The released ADP has the ability to greatly amplify and stabilize platelet aggregation. The evidence is mounting that agonist-induced platelet activation signals also crosstalk with integrin outside-in signals to regulate platelet responses [25]. The interaction between secreted ADP and its receptor P2Y(12) has also been shown to play a critical role in the sustained $\alpha \mathrm{IIb} / \beta 3$ activation [27]. To explore whether or not the inhibitory effect of GBE is dependent on the released ADP, we applied apyrase, which can diphosphohydrolyze ADP. Collagen-induced and U46619-induced platelet aggregation, both largely dependent on ADP secretion, were significantly reduced by apyrase; the combination of apyrase and GBE further enhanced the inhibition and virtually abolished collagen-induced and U46619-induced platelet aggregation [7]. This result suggests that GBE can also directly inhibit downstream molecules of U46619 and collagen.

\section{Conclusion}

GBE inhibited platelet activation mainly through the inhibition of Akt phosphorylation. Regarding the key role of Akt in regulating platelet activation and subsequent thrombosis development, our study results support the view that GBE may become a promising antithrombotic therapeutic agent that specifically targets the Akt-dependent molecular signaling pathway.

\section{References}

$\checkmark 1$ Cosemans JM, Munnix IC, Wetzker R, et al: Continuous signaling via PI3K isoforms $\beta$ and $\gamma$ is required for platelet ADP receptor function in dynamic thrombus stabilization. Blood 2006;108:3045-3052.

-2 Kharas MG, Okabe R, Ganis JJ, et al: Constitutively active AKT depletes hematopoietic stem cells and induces leukemia in mice. Blood 2010;115:1406-1415.

-3 Reheman A, Yang H, Zhu G, et al: Plasma fibronectin depletion enhances platelet aggregation and thrombus formation in mice lacking fibrinogen and von Willebrand factor. Blood 2009;113:1809-1817.

4 Yin B, Xu Y, Wei R, et al: Ginkgo biloba on focal cerebral ischemia: a systematic review and meta-analysis. Am J Chin Med 2014;42:769-783.

$5 \mathrm{Hu} \mathrm{H}, \mathrm{Li} \mathrm{N}$, Yngen M, et al: Enhanced leukocyte-platelet cross-talk in type 1 diabetes mellitus: relationship to microangiopathy. J Thromb Haemost 2004;2:58-64.

6 Otahbachi M, Simoni J, Simoni G, et al: Gender differences in platelet aggregation in healthy individuals. J Thromb Thrombolysis 2010;2:184-191.

7 Huang ZS, Zeng CL, Zhu LJ, et al: Salvianolic acid A inhibits platelet activation and arterial thrombosis via inhibition of phosphoinositide 3-kinase. J Thromb Haemost 2010;8:1383-1393.

8 Yin H, Stojanovic A, Hay N, et al: The role of Akt in the signaling pathway of the glycoprotein Ib-IX induced platelet activation. Blood 2008;111:658-665.

9 Gilio K, Munnix IC, Mangin P, et al: Non-redundant roles of phosphoinositide 3-kinase isoforms alpha and beta in glycoprotein VI-induced plateletsignaling and thrombus formation. J Biol Chem 2009;284:33750-33762. 
Halil M, Cankurtaran M, Yavuz BB, et al: No alteration in the PFA-100 in vitro bleeding time induced by the Ginkgo biloba special extract, EGb 761, in elderly patients with mild cognitive impairment. Blood Coagul Fibrinolysis 2005; 16:349-353.

$\checkmark 11$ Kohler S, P Funk, M Kieser: Influence of a 7-day treatment with Ginkgo biloba special extract EGb 761 on bleeding time and coagulation: a randomized, placebo-controlled, double-blind study in healthy volunteers. Blood Coagul Fibrinolysis 2004;15:303-309.

12 Ryu KH, Han HY, Lee SY, et al: Ginkgo biloba extract enhances antiplatelet and antithrombotic effects of cilostazol without prolongation of bleeding time. Thromb Res 2009;124:328-334.

13 Peng CC, Glassman PA, Trilli LE, et al: Incidence and severity of potential drug-dietary supplement interactions in primary care patients. An exploratory study of 2 outpatient practices. Arch Intern Med 2004;164:630-636.

14 Bone KM: Potential interaction of Ginkgo biloba leaf with antiplatelet or anticoagulant drugs: what is the evidence? Mol Nutr Food Res 2008;52:764-771.

15 Radomski MW, Palmer RM, Moncada S: An l-arginine/nitric oxide pathway present in human platelets regulates aggregation. Proc Natl Acad Sci USA 1990;87:5193-5197.

16 Radomski MW, Rees DD, Dutra A, et al: S-nitroso-glutathione inhibits platelet activation in vitro and in vivo. Br J Pharmacol 1992;107:745-749.

17 Freedman JE, Loscalzo J, Benoit SE, et al: Decreased platelet inhibition by nitric oxide in two brothers with a history of arterial thrombosis. J Clin Invest 1996;97:979-987.

18 Hynes RO: Integrins: versatility, modulation, and signaling in cell adhesion. Cell 1992;69:11-25.

19 Miranti CK, Leng L, Maschberger P, et al: Identification of a novel integrin signaling pathway involving the kinase Syk and the guanine nucleotide exchange factor Vav1. Curr Biol 1998;8:1289-1299.

20 Daniel J L, Dangelmaier CA, Mada S, et al: Cbl-b is a novel physiologic regulator of glycoprotein VI-dependent platelet activation. J Biol Chem 2010;285:17282-17291.

21 Bauer M, Maschberger P, Quek L, et al: Genetic and pharmacological analyses of involvement of Src-family, Syk and Btk tyrosine kinases in platelet shape change. Src-kinases mediate integrin alphaIIb beta3 inside-out signalling during shape change. Thromb Haemost 2001;8:331-340.

$>22$ Judd BA, Myung PS, Leng L, et al: Hematopoietic reconstitution of SLP-76 corrects hemostasis and platelet signaling through alpha IIb beta 3 and collagen receptors. Proc Natl Acad Sci USA 2000;97:12056-12061.

23 Watson SP, Auger JM, McCarty OJ, et al: GPVI and integrin alphaIIb beta3 signaling in platelets. J Thromb Haemost 2005;3:1752-1762.

-24 McCarty OJ, Larson MK, Auger JM, et al: Rac1 is essential for platelet lamellipodia formation and aggregate stability under flow. J Biol Chem 2005;280:39474-39484.

25 Kamae T, Shiraga M, Kashiwagi H, et al: Critical role of ADP interaction with P2Y12 receptor in the maintenance of alpha(IIb)beta3 activation: association with Rap1B activation. J Thromb Haemost 2006;4:13791387.

26 Li Z, Delaney MK, O’Brien KA, et al: Signaling during platelet adhesion and activation. Arterioscler Thromb Vasc Biol 2010;30:2341-2349.

27 Stojanovic A, Marjanovic JA, Brovkovych VM, et al: A phosphoinositide 3-kinase-AKT-nitric oxide-cGMP signaling pathway in stimulating platelet secretion and aggregation. J Biol Chem 2006;281:16333-16339. 